\title{
Alemtuzumab in the treatment of multiple sclerosis: patient selection and special considerations
}

\author{
This article was published in the following Dove Press journal: \\ Drug Design, Development and Therapy \\ 18 October 2016 \\ Number of times this article has been viewed
}

\author{
Jan Dörr ${ }^{1,2}$ \\ Karl Baum \\ 'Multiple Sclerosis Center, Neurology \\ Department, Klinik Hennigsdorf, \\ Hennigsdorf, ${ }^{2}$ NeuroCure Clinical \\ Research Center, Charité - \\ Universitätsmedizin Berlin, \\ Berlin, Germany
}

\begin{abstract}
Multiple sclerosis (MS) is among the most common chronic inflammatory diseases of the central nervous system. Although not curable, the constantly increasing armamentarium of disease-modifying drugs now allows control of disease activity in many patients. The humanized monoclonal antibody alemtuzumab is a powerful drug licensed for the treatment of MS. Upon binding to the CD52 surface protein on $\mathrm{CD}^{+}$and $\mathrm{CD} 8^{+} \mathrm{T}$ cells, $\mathrm{B}$ cells, and monocytes, circulating $\mathrm{CD}_{2} 2^{+}$cells are eliminated via antibody- and complement-mediated lysis, and a less autoreactive adaptive immune system is reconstituted. The efficacy of alemtuzumab in terms of both clinical and magnetic resonance imaging outcomes has been demonstrated in several phase II/III trials including long-term extensions and follow-up studies. Treatment response to alemtuzumab is strongest as long as active inflammation is the predominant pathophysiological feature, and it is becoming less efficacious in neurodegeneration-dominated later stages of the disease. Thus, the optimal placement of alemtuzumab within treatment algorithms of MS is crucial. The impressive efficacy of alemtuzumab is counteracted by a less favorable safety profile. Besides usually manageable infusion-associated side effects, development of secondary autoimmunity in almost half of treated patients is the most disconcerting risk of alemtuzumab. The high frequency, the delayed occurrence, and the potentially severe course of secondary autoimmune diseases require awareness and a close long-term monitoring of patients treated with alemtuzumab. Biomarkers that would allow prediction of treatment response to alemtuzumab on the one hand and identification of patients at risk for the development of secondary autoimmune diseases on the other are not yet available. Thus, the overall success of alemtuzumab treatment critically depends on the patient selection. The aim of this article is therefore, to characterize the significance of alemtuzumab in the treatment of MS with a focus on the selection of the optimal patient.
\end{abstract}

Keywords: multiple sclerosis, treatment, safety, efficacy, selection, benefit risk relation

\section{Introduction}

Multiple sclerosis (MS) is the most common chronic inflammatory disease of the central nervous system (CNS) in western countries and the leading cause of nontraumatic neurological disability in young adults. Although still not curable, disease activity can now be controlled in many patients by a variety of disease-modifying drugs (Table 1). However, modern drug treatment of MS is facing a dilemma: on the one hand, the armamentarium of available drugs is constantly increasing, yet on the other, there is an unmet need of evidence-based guidance on choosing the optimal treatment for the individual patient. ${ }^{1}$ The lack of valid predictive biomarkers for both treatment response and risk of side effects on the patient level is reinforced by the fact that potency and
NeuroCure Clinical Research Center,

Charité - Universitätsmedizin Berlin,

Charitéplatz I, I0II7 Berlin, Germany

Tel +493302545 4729

Email jan-markus.doerr@charite.de 
Table I Disease-modifying drugs approved for multiple sclerosis

\begin{tabular}{|c|c|c|c|}
\hline Drug & Application & Mode of action & Common side effects \\
\hline Interferon beta-Ia/Peginterferon & Intramuscular/ & Pleiotrophic & Flu-like symptoms, injection-site \\
\hline beta-Ia/ Interferon beta-Ib & subcutaneous & immunomodulatory cytokine & reactions, hepatopathy \\
\hline Glatiramer acetate & Subcutaneous & Immunomodulatory polypeptide & Injection-site reactions, lipodystrophy \\
\hline Teriflunomide & Oral & $\begin{array}{l}\text { Reversible inhibition of } \\
\text { dihydroorotate-dehydrogenase }\end{array}$ & $\begin{array}{l}\text { Hepatopathy, gastrointestinal side } \\
\text { effects, hair thinning }\end{array}$ \\
\hline Dimethylfumarate & Oral & $\begin{array}{l}\text { Activation of NrF } 2 \text { pathway (?), } \\
\text { antioxidant (?) }\end{array}$ & $\begin{array}{l}\text { Flush syndrome, gastrointestinal side } \\
\text { effects, lymphopenia }\end{array}$ \\
\hline Fingolimod & Oral & $\begin{array}{l}\text { Sphingosine-I-phosphate } \\
\text { receptor modulator }\end{array}$ & $\begin{array}{l}\text { Infections, hepatopathy, } \\
\text { bradyarrhythmia, gastrointestinal side } \\
\text { effects }\end{array}$ \\
\hline Natalizumab & Intravenous & mAb against $\alpha 4 \beta$ I-integrine & $\begin{array}{l}\text { Opportunistic infections, } \\
\text { gastrointestinal side effects, infusion- } \\
\text { associated reactions }\end{array}$ \\
\hline Alemtuzumab & Intravenous & $\mathrm{mAB}$ against $\mathrm{CD} 52$ & $\begin{array}{l}\text { Infections, infusion-associated } \\
\text { reactions, secondary autoimmune } \\
\text { disorders }\end{array}$ \\
\hline Daclizumab & Subcutaneous & $\mathrm{mAB}$ against $\mathrm{CD} 25$ & $\begin{array}{l}\text { Infections, skin reactions, } \\
\text { hepatopathy }\end{array}$ \\
\hline Mitoxantrone & Intravenous & $\begin{array}{l}\text { DNA intercalation, inhibition of } \\
\text { DNA/RNA synthesis }\end{array}$ & $\begin{array}{l}\text { Infections, gastrointestinal side } \\
\text { effects, myelopsuppression, } \\
\text { cardiotoxicity, secondary neoplasia }\end{array}$ \\
\hline
\end{tabular}

Note: "?" Highlights the hypothetical character of these risk factors.

Abbreviations: DNA, deoxyribonucleic acid; mAB, monoclonal antibody; NrF 2, nuclear factor 2 related factor; RNA, ribonucleic acid.

safety of a drug are usually inversely related, meaning that the more powerfully a drug suppresses disease activity the more severe safety and tolerability issues need to be considered. For the treatment of MS two not necessarily exclusive treatment paradigms are currently discussed: induction therapy advocating the early use of the most potent drugs and accepting a less favorable safety and tolerability profile to allow for maximum disease control from earliest disease phase on versus escalation therapy promoting safer and more tolerable but less effective drugs for the initial treatment and stepping-up as the disease progresses. ${ }^{2}$ Alemtuzumab is among the most potent currently available drugs for disease modification in MS and a candidate for both induction and escalation strategies. The aim of this article is to characterize the significance of alemtuzumab in the treatment of MS with a focus on the selection of the optimal patient.

\section{Alemtuzumab in multiple sclerosis Pharmacodynamics}

Alemtuzumab is a humanized monoclonal antibody against the cell surface protein CD52 which is primarily expressed on $\mathrm{CD}^{+}$and $\mathrm{CD}^{+}{ }^{+}$lymphocytes, $\mathrm{B}$ cells, and monocytes. The physiological role of CD52 is not known. Upon binding, alemtuzumab rapidly and effectively eliminates circulating $\mathrm{CD}_{2}{ }^{+}$cells via antibody- and complement-mediated depletion. ${ }^{3,4}$ Shortly after application the peripheral blood is virtually devoid of circulating lymphocytes. $\mathrm{CD} 52^{+}$cells in the lymphoid organs are less affected. Subsequently, the adaptive immune system reconstitutes from precursor cells or mature cells that have escaped depletion. The dynamics of repopulation differ in the respective cell lineages: monocytes and B cells are the first to reappear in the peripheral blood $\sim 3-6$ months after treatment. T cells, particularly $\mathrm{CD} 4^{+}$ cells repopulate substantially slower, reaching normal levels after $\sim 2-3$ years and pretreatment levels only after $\sim 5$ years. ${ }^{4-6}$ As regulatory $\mathrm{T}$ cells repopulate more quickly, these cells are relatively enriched in the peripheral blood which possibly constitutes a mode of action of alemtuzumab. The dynamics of the repopulation process and the relative reconstitution from either precursor cells or escaped cells may be critical for the treatment effect on the individual patient level. ${ }^{7}$

\section{Clinical research program}

The component was initially developed in the 1970s for the treatment of chronic lymphatic leukemia. In the early 1990s, alemtuzumab was first tested in patients with MS. These initial studies showed complete suppression of relapses and development of new brain lesions in patients with relapsingremitting MS (RRMS) and secondary progressive MS (SPMS). However, SPMS patients continued to accumulate disability and brain atrophy whereas RRMS patients showed a substantial and sustained improvement of neurological function. ${ }^{6,8}$ Although rather disappointing in terms of efficacy in SPMS these initial clinical trial experiences set the stages 
for the now well-established theory that MS is a two-phase disease in which inflammatory processes in the first phase trigger neuroaxonal degeneration in the second phase. The neurodegenerative process may then progress independently, even when the inflammation is eventually controlled. The obvious conclusion from this perception was that a consequent suppression of inflammatory activity in the early disease phase may reduce neurodegeneration in the later phase, thereby preventing an accumulation of permanent disability and eventually improving the prognosis. ${ }^{6}$ The lesson learned from this was that powerful anti-inflammatory drugs like alemtuzumab need to be placed early within the treatment algorithms in MS; not necessarily as a first line treatment but clearly as long as inflammation is the predominant pathophysiological feature, and definitely not as a last resort option in late stage disease when all other disease-modifying strategies have failed. ${ }^{8}$ This concept was further corroborated by two open label studies showing a beneficial effect of alemtuzumab on relapses and disability in both treatment-naïve patients and nonresponders to interferon (IFN) beta., ${ }^{9,10}$ Subsequently, the focus was set on patients with ongoing relapses and/or activity on magnetic resonance imaging (MRI). The clinical research program comprises the phase II multicenter CAMMS223 trial which enrolled 334 treatment-naïve RRMS patients, the "Comparison of Alemtuzumab and Rebif Efficacy in Multiple Sclerosis, Study I" (CARE-MS I) which enrolled 581 treatment-naïve RRMS patients, and CARE-MS II which enrolled 840 RRMS patients who had relapses while on disease-modifying therapy with mainly IFN beta or glatiramer acetate for at least 6 months. ${ }^{11-13}$ By inclusion criteria, patients in all studies had highly active disease defined by at least two relapses in the previous 2 years. In CAMMS223 at least one contrast enhancing lesion in 1 of 4 monthly baseline MRIs was additionally required. The mean disease duration from first onset to enrolment was $\sim 1.5$ years in CAMMS223 and CARE-MS I, and $\sim 4$ years in CARE-MS II without significant differences in the respective treatment arms. Baseline expanded disability status scale (EDSS) was 2.0 in CAMMS223 and CARE-MS I, and 2.5 in CARE-MS II. The treatment strategy reflects early aggressive treatment in highly active disease. Of note, all three studies tested efficacy of alemtuzumab against IFN beta- $1 \mathrm{a}, 44 \mu \mathrm{g}$ three times a week. Placebo-controlled trials have not been done. The coprimary endpoints were relapse rate and time to sustained accumulation of disability. Alemtuzumab was administered at a fixed dose of $12 \mathrm{mg}$ on 5 consecutive days followed by a second course over 3 days 12 months later. The initially started evaluation of a $24 \mathrm{mg}$ dose was discontinued later. The follow-up periods were 36 months in CAMMS223 and 24 months in CARE-MS I and CARE-MS II. Patients completing one of the three studies were eligible to participate in an open label extension study. Retreatment with $12 \mathrm{mg}$ over 3 consecutive days was possible at least 12 months after the last course, if necessary. According to long-term observations, about half of the patients require retreatment. ${ }^{14}$ For CAMMS223 5- and 10-year follow-up efficacy and safety data are available. ${ }^{15,16}$ The extension studies of the other two trials are still ongoing. Interim data have recently been reported. ${ }^{17,18} \mathrm{~A}$ recent Cochrane analysis rated the overall methodological quality of the three studies as moderate to high. ${ }^{19}$

\section{Efficacy and safety}

An extensive review of the study results is beyond the scope of this article and has been published elsewhere. ${ }^{20,21}$ In summary, relapse rates were substantially reduced in both alemtuzumab and IFN beta treatment arms. Interestingly, the relapse rate in the IFN beta arm of CARE-MS II also dropped from 1.5 in the year before study participation to 0.5 in year 2 despite remaining on IFN beta or switching from glatiramer acetate. Across the three studies, alemtuzumab significantly reduced relapse rates relative to high dose IFN beta-1a by $69 \%$ and $55 \%$ in the first-line treated patients (CAMMS223 and CARE-MS-I) and $49 \%$ in the second-line treated patients (CARE-MS-II). The proportion of relapse-free patients was significantly higher in the alemtuzumab groups compared to the IFN beta groups. It is important to realize that 20\%-35\% of alemtuzumab-treated patients continued to have relapses. ${ }^{20}$ In a recently published multicenter long-term follow-up of 100 alemtuzumab-treated patients, 40\% required at least one additional treatment course. ${ }^{22}$ In both CAMMS223 and CARE-MS II the proportion of patients with sustained accumulation of disability was significantly lower in the alemtuzumab arms (CAMMS223: alemtuzumab 9\%; IFN beta 26\%; $P<0.001 ; 71 \%$ risk reduction; CARE-MS II: alemtuzumab $9 \%$; IFN beta $22 \% ; P=0.0002 ; 59 \%$ risk reduction). In fact, in both studies patients treated with alemtuzumab slightly improved on the EDSS while IFN beta-treated patients deteriorated. CARE-MS I, however, failed to detect a superiority of alemtuzumab in preventing sustained accumulation of disability (alemtuzumab 8\%; IFN beta $11 \%, P=0.2$ ) which is probably due to the unexpected low disability progression in the IFN group. In fact, both arms showed a slight EDSS improvement. ${ }^{20}$ In the 5-year extension and the 10 -year follow-up of CAMMS223 relapse rates remained low, and EDSS remained stable or slightly improved in the vast majority of patients, although mean EDSS slightly increased. ${ }^{15,16}$ 
Improvement upon alemtuzumab treatment was, however, not corroborated in another cohort with a mean follow-up of 6.1 years. $^{22}$

The effect of alemtuzumab on MRI endpoints has mainly been addressed in the CARE-MS studies. Compared to IFN beta, alemtuzumab significantly reduced the proportion of patients with contrast enhancing $\mathrm{T} 1$ lesions by $53 \%$ in CARE-MS I and 61\% in CARE-MS II. Less impressive but still statistically significant was the reduction of the proportion of patients with new or enlarging T2 hyperintense lesions (48\% vs 58\% in CARE-MS I; $46 \%$ vs $68 \%$ in CARE-MS II). The post hoc determination of the compound measure "no evidence of disease activity" (NEDA), which integrates freedom of relapses, freedom of disability progression, and freedom of new or enlarging MRI lesions revealed a significantly increased proportion of the NEDA status in the alemtuzumab arms (CARE-MS I: alemtuzumab 39\%, IFN 27\%; $P=0.006$; CARE-MS II: alemtuzumab 32\%, IFN $14 \% ; P<0.0001) .{ }^{20}$

The sustained low relapse rates and the stability or even improvement of neurological function in the 5-year CAMMS223 extension study and the 10-year follow-up indicate a long-term effect of alemtuzumab and support the concept of early aggressive treatment. ${ }^{15,16}$ Furthermore, in the long-term follow-up of CARE-MS I over $60 \%$ of patients with NEDA status in year 2 who received no further treatment following the initial two courses maintained NEDA through year $5 .{ }^{17}$ But, about up to one-third of patients show an insufficient response to treatment, and over the longer run a third treatment course is necessary in $\sim 40 \%$ and a 4 th or 5 th course in $\sim 10 \%$ of patients. ${ }^{15,16,22}$ Postauthorization data are necessary to evaluate the long-term effect in a real world setting.

The obvious flip side of the impressive efficacy in the majority of patients is the occurrence of potentially hazardous side effects, although the proportion of serious adverse events in CAMMS223, CARE-MS I, and CARE-MS II did not differ significantly in the treatment arms. ${ }^{1-13}$ Across all studies, infusion-associated reactions, believed to derive from cytokine release upon cell lysis, were the most common side effects with an overall incidence of $>90 \%$. Infusion-associated reactions mainly comprise headaches, rash, pyrexia, nausea, urticaria, and fatigue, are usually mild to moderate, considered serious in $\sim 3 \%$, and can be controlled by glucocorticoid infusions as part of the treatment protocol. ${ }^{23}$ As can be expected from the profound depletion of lymphocytes, infections particularly of the respiratory and urinary tracts may occur. The risk of infection peaks during the first year of treatment and declines thereafter. ${ }^{15,16}$ Infections are mostly mild to moderate and can be handled with standard care. Nonetheless, several reports of opportunistic infections such as listeria meningitis and cerebral nocardiosis highlight the importance of an increased awareness. ${ }^{13,24}$ After an increase of herpesvirus infections was observed in the pivotal studies, prophylactic treatment with aciclovir was implemented in the study protocols and is now part of the risk management plan. Although several cases of progressive multifocal leukoencephalopathy (PML) have been reported in patients receiving alemtuzumab for other conditions and in the context of prior immunosuppression, ${ }^{25,26} \mathrm{PML}$ has not been reported in MS patients treated with alemtuzumab.

The most disconcerting risk attributable to alemtuzumabtreatment is autoimmunity, which is thought to arise from the dynamics and the clonal diversity of $\mathrm{T}$ cell repopulation. After depletion, $T$ cells repopulate either from new generation in the thymus or from proliferation of mature $T$ cells that have escaped depletion. The proportion of newly generated $\mathrm{T}$ cells and T cells proliferating from "old" surviving T cells seem to be predictive for the development of secondary autoimmune disorders, as alemtuzumab-treated patients with a higher proportion of newly generated $\mathrm{T}$ cells tend to have a lower risk of autoimmunity, probably because of the higher clonal diversity of the resulting $\mathrm{T}$ cell pool. ${ }^{27}$ Secondary autoimmune disorders may develop after alemtuzumab treatment, probably irrespective of the number of administered alemtuzumab courses. ${ }^{14}$ According to long-term data of the initial studies, almost half of the patients developed secondary autoimmunity over a follow-up of up to 10 years. ${ }^{14-16}$ Consistent to the course of $\mathrm{T}$ cell repopulation, the period with the highest risk of secondary autoimmunity is within 6-60 months after the first course with an incidence peak in the 3rd year and a decline in the years thereafter. ${ }^{15,28}$ The most relevant secondary autoimmune disorders affect the thyroid and include de novo development of hyperthyroidism, hypothyroidism, and thyroiditis. In the 5-year follow-up of CAMMS223, 39\% of alemtuzumab-treated patients developed autoimmune thyroid disease. ${ }^{15,28}$ Similar incidences of thyroid disorders have been observed in the 4-year follow-up of the CARE-MS studies. ${ }^{21}$

With an incidence of $2 \%$ across the clinical development program, idiopathic thrombocytopenic purpura (ITP) is a less common but potentially lethal autoimmune complication of alemtuzumab. In CAMMS223 one patient died from intracranial hemorrhage due to ITP, which prompted the implementation of monthly full blood counts in the risk management plan. ITP occurred 1-48 months after the last dose 
with a mean onset at 16 months and was usually responsive to first-line treatment with corticosteroids, intravenous immunoglobulins, and platelet transfusion. ${ }^{21,29}$

Even less common are autoimmune nephropathies. Across roughly 1,500 patients treated in the Phases II and III studies, four alemtuzumab-treated MS patients developed glomerulonephritis 4-39 months after the last dose. ${ }^{11,12,15}$

The high frequency, the delayed occurrence, and the potentially severe course of secondary autoimmune diseases together with the usually good response to standard treatment upon early diagnosis require awareness and a close long-term monitoring of alemtuzumab-treated patients. Biomarkers that would allow the identification of patients at risk for the development of secondary autoimmune diseases are not yet available. Increased pretreatment serum levels of interleukin (IL)-21 have been suggested to predict the development of autoimmune disorders months to years after alemtuzumab treatment; however, currently available detection assays for IL-21 have been shown to have only limited utility. ${ }^{30,31}$ Thus, strict adherence to the mandatory risk management plan comprising monthly laboratory tests and quarterly clinical evaluations is crucial for the risk reduction of alemtuzumab treatment and makes great demands on the compliance of both patients and physicians.

Ongoing clinical/translational trials on the mechanisms of action, clinical outcomes, and identification of distinct immunological changes upon treatment with alemtuzumab (eg, NCT02419378, NCT01395316, and NCT02623946) may provide more information on predictive biomarkers in the near future.

\section{Identifying treatment candidates}

Five simple questions help to decide whether or not a MS patient is a candidate for alemtuzumab treatment (Figure 1).

1. Does the patient comply with the local formal approval of alemtuzumab, for example, with respect to age, disease course, and previous treatments?

2. Does the patient have a high a priori probability to benefit from alemtuzumab?

3. Does the patient have any particular risk factors for reduced tolerability or safety?

4. Do disease activity and prognosis in the patient justify a potentially hazardous treatment?

5. Is the patient reliable enough to adhere to the prolonged risk management program?

\section{Approval of alemtuzumab for treatment of MS}

Alemtuzumab is currently licensed for the treatment of MS in $>30$ countries, but the local legal practice may vary. The European Medical Agency approved alemtuzumab in 2013 for the treatment of adult RRMS patients with active disease, whereupon "activity" is poorly defined. In contrast, the US Food and Drug Administration initially denied approval because of concerns about the design of the pivotal studies. In 2014, the US Food and Drug Administration eventually approved alemtuzumab in patients with RRMS who had had an inadequate response to at least two disease-modifying drugs. In Canada, alemtuzumab is approved for the management of adult RRMS patients with active disease defined by clinical and imaging features, and an inadequate response to

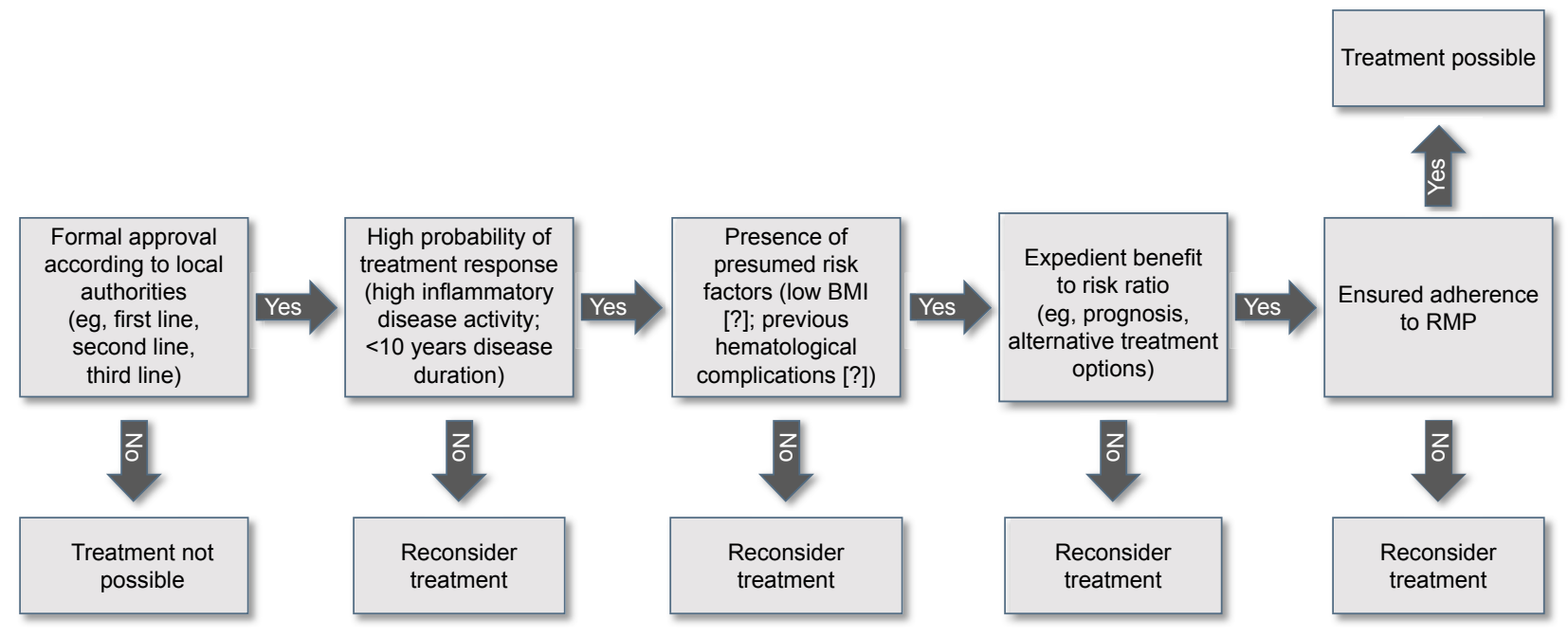

Figure I Algorithm for selection of patients eligible for alemtuzumab treatment.

Notes: The decision tree may help to decide whether a patient is a candidate for treatment with alemtuzumab. "?" Highlights the hypothetical character of these risk factors. Abbreviations: BMI, body mass index; RMP, risk management plan. 
IFN beta or other disease-modifying therapies. This means that in contrast to North America failure of previous diseasemodifying treatment is not required in European countries, and alemtuzumab can either be used as second-line option for treatment escalation and first-line option for induction of treatment. On the other hand, on-label treatment in the USA includes patients with SPMS who still have relapses whereas in Europe and Canada treatment is restricted to patients with relapsing-remitting disease. Pediatric use is approved in neither country.

\section{Probability of treatment response and risk of safety/tolerability problems}

As long as valid biomarkers for the prediction of treatment response before initiation of treatment are not available the probability to benefit from treatment remains to be deducted from the clinical trial data. The association of treatment response and extent of cerebral inflammation in the pretreatment phase suggests that treatment response critically depends on the presence of active inflammation. ${ }^{8}$ Thus, either or both continued relapses and radiographical activity are prerequisites in patients considered for alemtuzumab. For clinical routine, the definition of active disease in the CARE-MS studies ( $\geq 1$ relapse in the year before treatment and $\geq 2$ relapses in the past 2 years) and the definition of MRI activity in CAMMS223 ( $\geq 1$ contrast-enhancing lesion in 1 of 4 monthly baseline MRIs) may be used. In light of the rather short mean disease duration and low baseline EDSS in the study populations (1.3 years and EDSS 2.0 in CAMMS223 and 3.8 years and EDSS 2.5 in CARE-MS II) the decision for alemtuzumab should not be postponed to late stage disease. In conclusion, young patients with active disease and rather short disease duration have the highest probability of treatment response. Patients with secondary progressive disease are unlikely to benefit and should not be treated with alemtuzumab. Unfortunately, information on patients with longstanding disease are lacking since patients with disease duration $>10$ years have been excluded from the studies. The observation of comparable effects when alemtuzumab was used either in induction (CAMMS223 and CARE-MS I) or escalation (CARE-MS II) treatment paradigms indicates that alemtuzumab does not necessarily need to be given as a first line drug and in combination with the safety data rather argues for its use as an early escalation option.

The development of secondary autoimmunity is probably the most relevant risk associated with alemtuzumab, and a predictive marker to identify patients at risk for the development of autoimmune disorders would be a great step forward in the risk management of alemtuzumab. High serum levels of IL-21 might prove to be a predictor of autoimmune disease but measurement is still experimental and not yet recommended for clinical routine. ${ }^{30,31}$ An important yet unanswered question is whether the risk of secondary autoimmunity is increased in patients with additional preexisting autoimmune disease. Clinical experience suggests no exacerbation of autoimmune thyroid disease upon alemtuzumab treatment, but as long as systematic data are lacking a careful benefit-risk evaluation is necessary in patients with preexisting autoimmune conditions.

Several case reports suggest additional risk factors which obviously require further evaluation. According to a recent report of early onset ITP in two patients who both terminated previous fingolimod therapy because of severe lymphocytopenia, the development of lymphocytopenia under disease-modifying MS treatment may be a risk factor for the development of autoimmune thrombocytopenia under alemtuzumab. ${ }^{32}$ Another recent report of an opportunistic central nervous system nocardiosis infection in an anorectic patient (body mass index 14) 5 months after initiation of alemtuzumab treatment with prolonged lymphopenia raised the question of a general association between body mass index and safety of alemtuzumab. ${ }^{24}$ As long as this aspect has not been systematically evaluated, alemtuzumab should be used with caution in severely anorectic patients.

\section{Benefit-to-risk considerations}

Based on the concept of a window of opportunity for modulation of the long-term outcome via rigorous disease-modifying drug treatment the benefit-to-risk relation is obviously highest in patients who did not yet accumulate substantial permanent disability but are at high-risk to do so. ${ }^{33}$ The main challenge is now to identify these patients in the absence of markers that would allow a reliable prognosis on the level of the individual patient. Obviously, a patient with ongoing clinical and/ or MRI-supported inflammatory activity despite escalation therapy with natalizumab or fingolimod is a clear candidate for alemtuzumab. But would alemtuzumab also be justified without prior use of natalizumab or fingolimod? Several studies aimed to identify risk factors of a poor prognosis. On the group level, relapse frequency in the early disease phase, time to accumulation of moderate disability, age at disease onset, presence of oligoclonal cerebrospinal fluid bands, number of MRI lesions on the initial scan, and treatment effects on active MRI lesions all serve as surrogates for disability progression and thus poorer prognosis. ${ }^{34-38}$ 
As a rule of thumb that might be used to select patients for alemtuzumab, the more risk factors for poor outcome accumulate in a patient the more justifiable is an aggressive and potentially hazardous treatment.

Another situation that may argue for early alemtuzumab therapy is latent John Cunningham (JC) virus infection, indicated by a positive JC virus antibody test. In a JC virus antibody positive patient with active disease despite first-line therapy in which fingolimod is not a treatment option the risk of developing natalizumab-associated PML has to be balanced against the alemtuzumab-associated risks. In such a patient, early initiation of alemtuzumab treatment might be a superior option than natalizumab treatment for a very limited period.

Family planning might be another factor to consider in the benefit-risk evaluation. In a woman with active disease who wishes to become pregnant suppression of disease activity by a temporally defined alemtuzumab therapy might be a more acceptable option than continuous treatment with natalizumab or fingolimod, particularly since the latter needs to be suspended at least 3 months prior to conception.

\section{Adherence to the risk management program}

The prolonged risk management program of alemtuzumab, which comprises monthly whole blood counts and quarterly thyroid function tests over a period of 48 months after the last course is unique in MS therapy and challenging for both patients and physicians. It accommodates the observation of delayed development of secondary autoimmunity and was introduced after a fatal case of ITP in CAMMS223. ${ }^{13}$ The fact that complications may occur at any time reinforces the necessity of strict adherence to this program. ${ }^{14,32}$ The feasibility and expedience of the monitoring program have been demonstrated both in the clinical trial program and in clinical routine. ${ }^{22}$ In the progress of shared decision making the background and importance of the program should be explained to the patient who, in turn, must commit him- or herself to strictly adhere to the program. Alemtuzumab treatment should not be initiated in the case of serious doubts on the reliability of the patient.

\section{Conclusion}

Alemtuzumab is an important augmentation to the armamentarium of disease-modifying drugs for the treatment of MS. Efficacy is strongest in patients with highly active relapsing forms of the disease with predominantly inflammatory activity. In the escalation treatment concept the optimal position of alemtuzumab, irrespective of country-specific regulatory aspects, is early treatment escalation. Although data are sparse, it may also be used for induction therapy. Because of some severe but usually manageable safety and tolerability issues accurate patient selection is crucial. Since predictive efficacy and safety markers are not yet available, five simple questions have been suggested and elucidated here that may help in the clinical routine setting to identify eligible patients. Obviously, with each new drug licensed for MS therapy, the roles of alemtuzumab and all other disease-modifying drugs need to be reevaluated.

\section{Disclosure}

JD received research funding by Bayer Healthcare and Novartis, honoraria for lectures/advisory by Allergan, Bayer Healthcare, Biogen, Genzyme (Sanofi), Merck-Serono, Novartis, Teva, and travel support by Bayer Healthcare, Biogen, and Novartis.

$\mathrm{KB}$ received honoraria for lectures/advisory and travel support by Almirall, Bayer Healthcare, Biogen, Genzyme (Sanofi), Merck-Serono, Novartis, and Teva.

The authors report no other conflicts of interest in this work.

\section{References}

1. Teunissen CE, Malekzadeh A, Leurs C, Bridel C, Killestein J. Body fluid biomarkers for multiple sclerosis - the long road to clinical application. Nat Rev Neurol. 2015;11(10):585-596.

2. Wingerchuk DM, Carter JL. Multiple sclerosis: current and emerging disease-modifying therapies and treatment strategies. Mayo Clin Proc. 2014;89(2):225-240.

3. Jones DE, Goldman MD. Alemtuzumab for the treatment of relapsingremitting multiple sclerosis: a review of its clinical pharmacology, efficacy and safety. Expert Rev Clin Immunol. 2014;10(10):1281-1291.

4. Krumbholz M, Derfuss T, Hohlfeld R, Meinl E. B cells and antibodies in multiple sclerosis pathogenesis and therapy. Nat Rev Neurol. 2012;8(11):613-623.

5. Hill-Cawthorne GA, Button T, Tuohy O, et al. Long term lymphocyte reconstitution after alemtuzumab treatment of multiple sclerosis. J Neurol Neurosurg Psychiatry. 2012;83(3):298-304.

6. Coles AJ, Cox A, Le Page E, et al. The window of therapeutic opportunity in multiple sclerosis: evidence from monoclonal antibody therapy. J Neurol. 2006;253(1):98-108.

7. Cossburn MD, Harding K, Ingram G, et al. Clinical relevance of differential lymphocyte recovery after alemtuzumab therapy for multiple sclerosis. Neurology. 2013;80(1):55-61.

8. Coles AJ, Wing MG, Molyneux P, et al. Monoclonal antibody treatment exposes three mechanisms underlying the clinical course of multiple sclerosis. Ann Neurol. 1999;46(3):296-304.

9. Fox EJ, Sullivan HC, Gazda SK, et al. A single-arm, open-label study of alemtuzumab in treatment-refractory patients with multiple sclerosis. Eur J Neurol. 2012;19(2):307-311.

10. Hirst CL, Pace A, Pickersgill TP, et al. Campath 1-H treatment in patients with aggressive relapsing remitting multiple sclerosis. J Neurol. 2008;255(2):231-238.

11. Cohen JA, Coles AJ, Arnold DL, et al. Alemtuzumab versus interferon beta 1a as first-line treatment for patients with relapsing-remitting multiple sclerosis: a randomised controlled phase 3 trial. Lancet 2012;380(9856):1819-1828. 
12. Coles AJ, Twyman CL, Arnold DL, et al. Alemtuzumab for patients with relapsing multiple sclerosis after disease-modifying therapy: a randomised controlled phase 3 trial. Lancet. 2012;380(9856):1829-1839.

13. Coles AJ, Compston DA, Selmaj KW, et al. Alemtuzumab vs interferon beta-1a in early multiple sclerosis. N Engl J Med. 2008;359(17): 1786-1801.

14. Tuohy O, Costelloe L, Hill-Cawthorne G, et al. Alemtuzumab treatment of multiple sclerosis: long-term safety and efficacy. J Neurol Neurosurg Psychiatry. 2015;86(2):208-215.

15. Coles AJ, Fox E, Vladic A, et al. Alemtuzumab more effective than interferon $\beta$-1a at 5-year follow-up of CAMMS223 clinical trial. $\mathrm{Neu}$ rology. 2012;78(14):1069-1078.

16. Coles AJ, Habek M, Bass A, et al. Durable efficacy of alemtuzumab over 10 years: long-term follow-up of patients with RRMS from the CAMMS223 study. Neurology. 2016;86(16):P3.053.

17. Giovannoni G, Arnold DL, Cohen J, et al. Long-term responders from the CARE-MS I study: no evidence of disease activity for 4 years following 2 courses of alemtuzumab and no further treatment. Neurology. 2016;86(16):P3.054.

18. Wiendl H, Arnold DL, Cohen J, et al. No evidence of disease activity for 4 years following 2 courses of alemtuzumab and no further treatment: long-term responders from the CARE-MS II study. Neurology. 2016;86(16):P3.059.

19. Riera R, Porfírio GJ, Torloni MR. Alemtuzumab for multiple sclerosis. Cochrane Database Syst Rev. 2016;4:CD011203.

20. Menge T, Stüve O, Kieseier BC, Hartung H-P. Alemtuzumab: the advantages and challenges of a novel therapy in MS. Neurology. 2014; 83(1):87-97.

21. Havrdova E, Horakova D, Kovarova I. Alemtuzumab in the treatment of multiple sclerosis: key clinical trial results and considerations for use. Ther Adv Neurol Disord. 2015;8(1):31-45.

22. Willis MD, Harding KE, Pickersgill TP, et al. Alemtuzumab for multiple sclerosis: Long term follow-up in a multi-centre cohort. Mult Scler J. 2015;22(9):1215-1223.

23. Caon C, Meyer C, Mayer L, Smith MS. Efficacy and safety of alemtuzumab in multiple sclerosis and impact on nursing role. Int J MS Care. 2013;15(4):159-168.

24. Penkert H, Delbridge C, Wantia N, Wiestler B, Korn T. Fulminant central nervous system nocardiosis in a patient treated with alemtuzumab for relapsing-remitting multiple sclerosis. JAMA Neurol. 2016;73(6):757-759.

25. Isidoro L, Pires P, Rito L, Cordeiro G. Progressive multifocal leukoencephalopathy in a patient with chronic lymphocytic leukaemia treated with alemtuzumab. BMJ Case Rep. 2014.
26. Waggoner J, Martinu T, Palmer SM. Progressive multifocal leukoencephalopathy following heightened immunosuppression after lung transplant. J Heart Lung Transplant. 2009;28(4):395-398.

27. Jones JL, Thompson SAJ, Loh P, et al. Human autoimmunity after lymphocyte depletion is caused by homeostatic T-cell proliferation. Proc Natl Acad Sci US A. 2013;110(50):20200-20205.

28. Daniels GH, Vladic A, Brinar V, et al. Alemtuzumab-related thyroid dysfunction in a phase 2 trial of patients with relapsing-remitting multiple sclerosis. J Clin Endocrinol Metab. 2014;99(1):80-89.

29. Cuker A, Stasi R, Palmer J, Oyuela P-, Margolin DH, Bass A. Successful detection and management of immune thrombocytopenia in alemtuzumab-treated patients with active relapsing-remitting multiple sclerosis. Neurology. 2014;82(10 Suppl):P2.198.

30. Azzopardi L, Thompson SAJ, Harding KE, et al. Predicting autoimmunity after alemtuzumab treatment of multiple sclerosis. J Neurol Neurosurg Psychiatry. 2014;85(7):795-798.

31. Jones JL, Phuah C-L, Cox AL, et al. IL-21 drives secondary autoimmunity in patients with multiple sclerosis, following therapeutic lymphocyte depletion with alemtuzumab (Campath-1H). J Clin Invest. 2009;119(7):2052-2061.

32. Obermann M, Ruck T, Pfeuffer S, Baum J, Wiendl H, Meuth SG. Simultaneous early-onset immune thrombocytopenia and autoimmune thyroid disease following alemtuzumab treatment in relapsing-remitting multiple sclerosis. Mult Scler. 2016;22(9):1235-1241.

33. Leray E, Yaouanq J, Le Page E, et al. Evidence for a two-stage disability progression in multiple sclerosis. Brain J Neurol. 2010;133(Pt 7): 1900-1913.

34. Tintore M, Rovira À, Río J, et al. Defining high, medium and low impact prognostic factors for developing multiple sclerosis. Brain. 2015; 138(Pt 7):1863-1874.

35. Scalfari A, Neuhaus A, Degenhardt A, et al. The natural history of multiple sclerosis: a geographically based study 10: relapses and longterm disability. Brain J Neurol. 2010;133(Pt 7):1914-1929.

36. Scalfari A, Neuhaus A, Daumer M, Ebers GC, Muraro PA. Age and disability accumulation in multiple sclerosis. Neurology. 2011;77(13): 1246-1252.

37. Sormani MP, Arnold DL, De Stefano N. Treatment effect on brain atrophy correlates with treatment effect on disability in multiple sclerosis. Ann Neurol. 2014;75(1):43-49.

38. Wattjes MP, Rovira À, Miller D, et al. Evidence-based guidelines: MAGNIMS consensus guidelines on the use of MRI in multiple sclerosis - establishing disease prognosis and monitoring patients. Nat Rev Neurol. 2015;11(10):597-606.
Drug Design, Development and Therapy

\section{Publish your work in this journal}

Drug Design, Development and Therapy is an international, peerreviewed open-access journal that spans the spectrum of drug design and development through to clinical applications. Clinical outcomes, patient safety, and programs for the development and effective, safe, and sustained use of medicines are the features of the journal, which

\section{Dovepress}

has also been accepted for indexing on PubMed Central. The manuscript management system is completely online and includes a very quick and fair peer-review system, which is all easy to use. Visit http://www.dovepress.com/testimonials.php to read real quotes from published authors. 